\title{
Hardware-in-the-loop simulation of wave energy converters based on dielectric elastomer generators
}

\author{
Giacomo Moretti $(\mathbb{D}) \cdot$ Andrea Scialò $(\mathbb{B}) \cdot$ Giovanni Malara $(\mathbb{D}) \cdot$ Giovanni Gerardo Muscolo $(\mathbb{D})$ \\ Felice Arena (1) - Rocco Vertechy (i) - Marco Fontana $(\mathbb{D}$
}

Received: 28 March 2020 / Accepted: 2 February 2021 / Published online: 26 February 2021

(C) The Author(s) 2021

\begin{abstract}
Dielectric elastomer generators (DEGs) are soft electrostatic generators based on low-cost electroactive polymer materials. These devices have attracted the attention of the marine energy community as a promising solution to implement economically viable wave energy converters (WECs). This paper introduces a hardware-in-the-loop (HIL) simulation framework for a class of WECs that combines the concept of the oscillating water columns (OWCs) with the DEGs. The proposed HIL system replicates in a laboratory environment the realistic operating conditions of an OWC/DEG plant, while drastically reducing the experimental burden compared to wave tank or sea tests. The HIL simulator is driven by a closed-loop real-time hydrodynamic model that is based on a novel coupling criterion which allows
\end{abstract}

G. Moretti · M. Fontana

PERCRO Laboratory, Scuola Superiore Sant'Anna, 56127 Pisa, Italy

G. Moretti

Intelligent Material Systems Laboratoy, Saarland

University, 66121 Saarbrücken, Germany

A. Scialò · G. Malara · F. Arena

Natural Ocean Engineering Laboratory (NOEL),

Mediterranea University of Reggio Calabria,

89122 Reggio Calabria, Italy

G. G. Muscolo

Department of Mechanical and Aerospace Engineering,

Politecnico di Torino, 10129 Turin, Italy rendering a realistic dynamic response for a diversity of scenarios, including large scale DEG plants, whose dimensions and topologies are largely different from those available in the HIL setup. A case study is also introduced, which simulates the application of DEGs on an OWC plant installed in a mild real sea laboratory test-site. Comparisons with available real sea-test data demonstrated the ability of the HIL setup to effectively replicate a realistic operating scenario. The insights gathered on the promising performance of the analysed OWC/DEG systems pave the way to pursue further sea trials in the future.

Keywords Smart materials - Dielectric elastomers Energy harvesting $\cdot$ Hardware in the loop

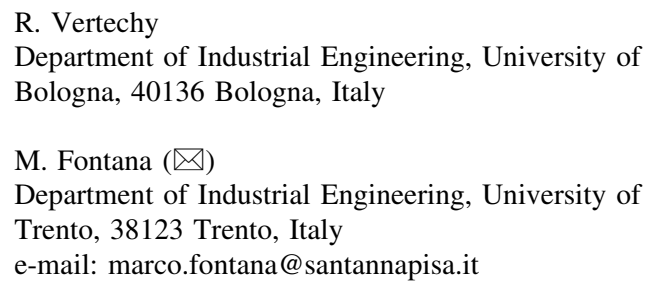




\section{Introduction}

Ocean wave power is among the most abundant resources of clean renewable energy and it has the potential to become one of the key contributors in the future energy mix. Nevertheless, wave energy technologies are struggling to reach industrial readiness, because of the high costs and stringent reliability requirements involved in the deployment of wave energy converter (WEC) plants [1].

In the last decade, the technology of dielectric elastomers (DEs) has become one of the potential game-changing solutions for wave energy applications $[2,3]$. DEs can be used to develop lightweight inexpensive electrostatic generators, namely DE generators (DEGs) [4, 5], which might provide power take-off (PTO) solutions for WECs featuring better adaptability to the highly variable and chemically aggressive sea environment compared to traditional hydraulic or electro-mechanical generators [6].

A WEC principle that appears to combine well with DEGs is the oscillating water column (OWC) [7, 8]. The OWC takes advantage of a canalized water volume subject to the wave loads to transfer mechanical energy towards a pneumatic chamber. Thus, pneumatically-driven DEGs [9] can be effectively employed as the PTO for OWCs. The DEG-based OWC has been largely investigated in the last few years [6]. Wave tank tests have been carried out to assess the energetic performance up to a scale of approximately 1:30 [10], and a first campaign of sea tests has been carried out in a mild natural basin to test the passive response (without electrical control) of a DEG-OWC in real waves [11].

In this paper, we present a hardware-in-the-loop (HIL) simulator which serves as a dry-run platform to test DEG prototypes and their power electronics, and faithfully simulates the dynamically coupled response of DEG-OWC plants. Compared to numerical models, the simulator offers an exact rendering of the DEG response, free from mathematical assumptions on the generator complex electro-visco-elastic behaviour. Compared to sea or tank tests, this HIL framework provides a drastic reduction in the costs and the operational risks.

The simulator consists of a software environment running a real-time hydrodynamic model of an OWC converter, and a mechanical interface that drives the physical DEG PTO deformation, emulating the action of an OWC. The software environment solves the equations of motion for an OWC taking into account the measured signals from the physical system. The mechanical interface is in turn driven based on the hydrodynamic model solution.

HIL simulators of WECs have been proposed in the past $([12,13]$ and [14]), primarily as a tool to investigate advanced PTO systems, whose employment in wave tank or at sea would be technically and/or economically prohibitive without prior laboratory scouting. In [12] and [13], hydraulic test rigs for HIL testing of the PTO system in heaving point-absorber WECs are proposed. In [14], a HIL setup to replicate the action of the wave loads on some functional components of a gyroscopebased WEC is presented. Application of HIL simulators for OWCs has been demonstrated in [15, 15], where large-scale laboratory testing of power take-off, control, and grid integration infrastructure has been pursued.

A very preliminary and limited demonstration of HIL simulation for DEG-based OWCs was proposed in [17] by some of the authors of this contribution. In that work, however, consistent coupling between the hydrodynamic OWC model and the physical DEG driving interface was not addressed. Here, in contrast, we propose for the first time coupling criteria which allow studying a diversity of scenarios, including the simulation of large DEG plants with several DEGs, whose dimensions generally differ from those of the tested physical samples. Moreover, compared to [17], the setup presented here marks a significant technological upscaling step, as it allows testing of Wattscale DEGs, consistently with the maximum scale reached to date by the DEG PTO technology.

The article is organized as follows. Section 2 shows an overview of the proposed HIL simulator and its components. Section 3 illustrates a relevant case study, involving a particular OWC topology known as U-OWC $[18,19]$. With reference to this application, we present tests in which a fully-functional DEG prototype is coupled with a hydrodynamic model of a scaled U-OWC [11], hence paving the way towards future sea tests on small-scale systems operating in a mild sea climate. Section 4 draws the conclusions.

\section{Hardware-in-the-loop testing of the DEG PTO for oscillating water columns}

The OWC WEC consists in a semi-submerged collector (either fixed or floating), partly open towards 

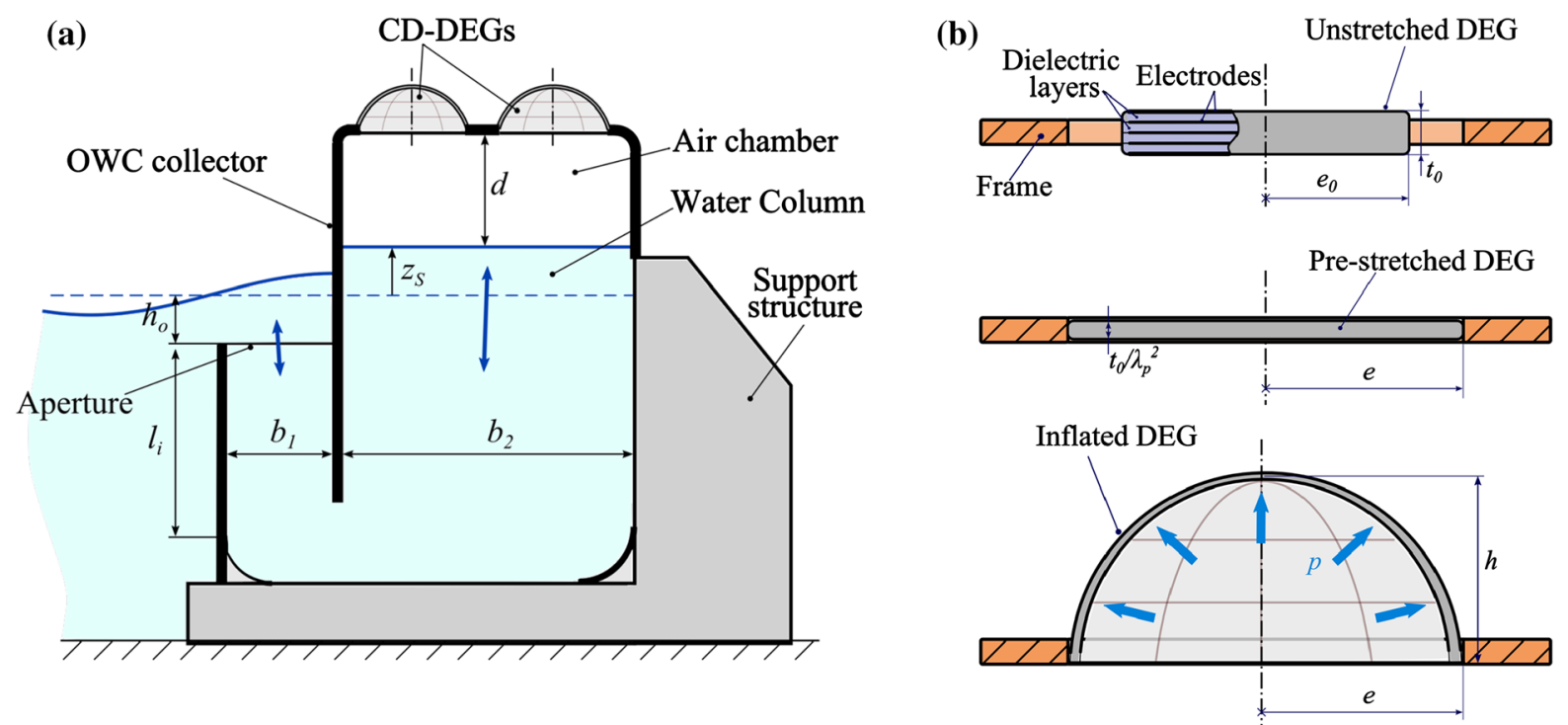

Fig. 1 a Schematic representation of an OWC (with U-shaped collector) holding a set of CD-DEGs as the PTO system. b Representation of a CD-DEG in the unstretched configuration

the sea, housing a water column which is put into oscillation by the incident waves (see Fig. 1a). Energy extraction is accomplished by exploiting the alternate compression and expansion of an air volume enclosed between the water column free surface and the collector (i.e., the air chamber). In a previous design, the pneumatic energy was converted into electrical energy by means of a bidirectional air turbine [8]. Recently, a special DEG layout, called a circular diaphragm DEG (CD-DEG), has been proposed as an alternative PTO system [6], which might allow overcoming problems related to the limited adaptability of the turbines to the harsh sea environment and their poor efficiency in the presence of bidirectional flows.

The CD-DEG (Fig. 1b) is a pre-stretched multilayer structure made of a set of DE layers separated by compliant electrodes with alternate polarity, so as to form a deformable capacitor [6]. The multi-layer structure allows applying large electric fields and, hence, the conversion of large energy densities while limiting the operating voltage. The stack is secured on a circular rigid frame with pre-stretch $\lambda_{p}=e / e_{0}$, where $e$ is the radius of the frame and $e_{0}$ is the radius of the unstretched stack. Upon pre-stretching, the unstretched stack thickness $t_{0}$ reduces to $t_{0} / \lambda_{p}^{2}$ (in the flat configuration) owing to the elastomer incompressibility. The application of a pressure difference (top), flat pre-stretch configuration (middle), and inflated configuration (bottom)

on the CD-DEG faces results in a bubble-like expansion of the deformable layers, i.e., an increase in the electrodes area, a decrease in the dielectric layers thickness and, hence, an increase in capacitance.

In OWCs with DEG PTO, a set of CD-DEGs are mounted on top of the collector, so as to isolate the air chamber volume from the atmosphere (Fig. 1a). The air chamber compressions/ expansions cause alternate outward/inward deformations of the CD-DEG. Cyclic DEG deformations can, in turn, be exploited to convert the mechanical work done by the air pressure into electrical energy, upon appropriate control of the CDDEG's voltage [6].

In order to realistically simulate the operation of an OWC/DEG in a laboratory environment, we hereby present a HIL system that combines: a hardware scaled implementation of the OWC air chamber and the DEG PTO, a mechanical hardware which emulates the action of the water column on the chamber, and a software environment which simulates the OWC hydrodynamic behaviour and executes the DEG's electrical control logics.

A block-diagram showing the elements and the variables flow in HIL simulations is shown in Fig. 2a. The main components of the HIL simulator are: (1) a fully-functional CD-DEG PTO that includes the elastomeric unit, the driving electronics and the sensing system; (2) a mechanical driving system 
(a)

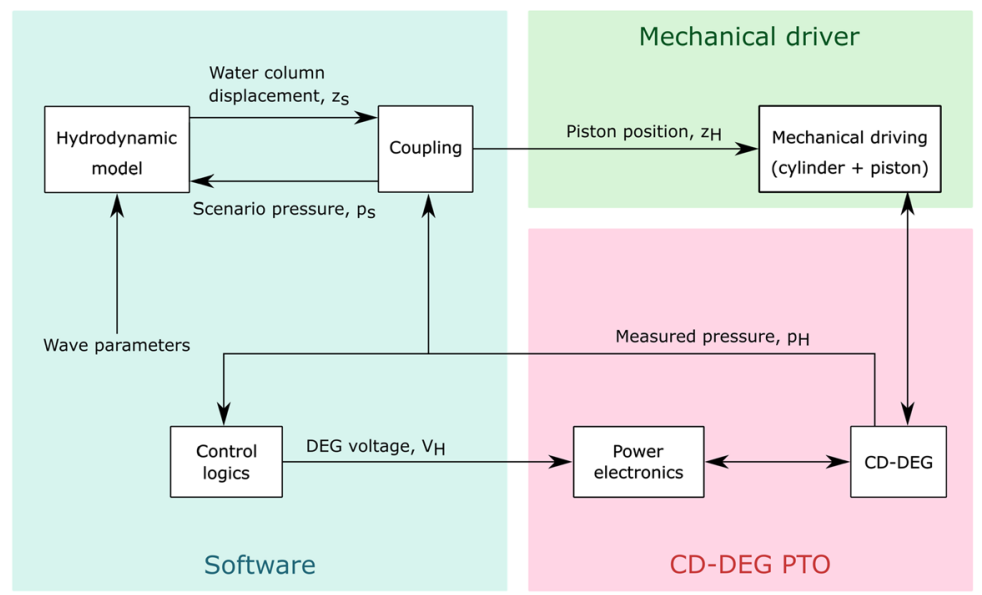

(b)

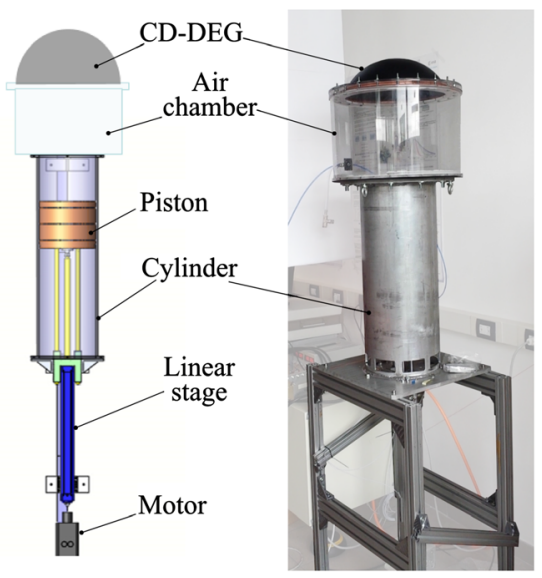

Fig. 2 a Block-diagram of HIL simulations combining a software hydrodynamic model of an OWC and a physical model of the air chamber-DEG assembly. b Schematic drawing and picture of the experimental setup for HIL testing

composed by a scaled hardware model of an OWC air chamber and position-controlled piston-cylinder set, which emulates the effect of the water column oscillations on the air chamber; (3) a software environment that solves in real-time the equations of motion for a reference OWC based on a hydrodynamic model and on the instantaneous readings of sensors, and controls the CD-DEG power electronics.

The hydrodynamic model receives the wave parameters as the simulation input, computes the wave loads, and solves the OWC equation of motion in real-time. The simulated displacement of the water column inside the OWC chamber is used to command the motion of the hardware piston. The relative air pressure measured in the cylinder is, in turn, fed back into the model and its contribution is accounted for in the equation of motion. The voltage applied on the DEG through the power electronics is controlled as a function of the measured control variables (here, the pressure on the CD-DEG). A coupling block is responsible for introducing corrections on the measured pressure signals and commanded piston displacements needed to guarantee a consistent coupled dynamic behaviour of the hardware and the simulated environment. Thus, the proposed HIL architecture potentially allows for the simulation of different scenarios with different OWC geometries and CDDEG with dimensional features which differ from those of the physical prototype.
In the following subsections, the main components of the HIL simulator are briefly described.

\subsection{Hydrodynamic model}

A nonlinear lumped-parameter hydrodynamic model is used for describing the OWC oscillations in the time domain. This modelling approach is widely used in WEC modelling, as suggested, for instance, in [18] and [19]. The model describes the water column oscillations via the unsteady Bernoulli equation. This approach is applied under the assumptions that the OWC collector is fixed and the water column width and breadth are small compared to the wavelength, so that the water column displacement is approximated by the oscillation of a rigid vertical piston. We further assume that the total head at the OWC inlet is given by the wave pressure head, thus neglecting other contributions. The wave field in front of the structure is described by the potential flow theory. Specifically, the calculation of the wave pressure head is accomplished in the framework of the linear potential wave theory [20]. The inlet wave pressure at the OWC collector aperture is calculated via the so-called Cummins equation [21], which accounts for the effects of the hydrodynamic memory and the hydrodynamic added mass. The equation governing the water column dynamics [19] is: 


$$
\begin{aligned}
& {\left[M\left(z_{S}\right)+A(\infty)\right] \ddot{z}_{S}+C\left(z_{S}, \dot{z}_{S}\right) \dot{z}_{S}} \\
& \quad+\frac{1}{g} \int_{-\infty}^{t} K(\tau) \dot{z}_{S}(t-\tau) d \tau+z_{S}+\frac{1}{\rho g} p_{S} \\
& \quad=\frac{1}{\rho g} \Delta p^{(D)}
\end{aligned}
$$

where $p_{S}$ is the relative pressure in the air chamber; $A(\infty)$ is the infinite-frequency added mass; $K(t)$ is called the retardation function; $M\left(z_{S}\right)$ is the timevarying inertia of the water column within the collector, which includes the effect of mass transfer between the water column and the wave field: it depends on the instantaneous water column level $z_{S}$ (i.e. the upward elevation with respect to the mean water level) and the OWC collector geometry; finally, $C\left(z_{S}, \dot{z}_{S}\right)$ is a damping coefficient associated with the kinetic head of the water column, which depends on the water column position and velocity and on the system geometry [19]. Commonly, the calculation of the hydrodynamic parameters $(A(\infty), K(t))$ and the excitation is pursued via boundary-element codes [22], although analytical solutions are available for some specific geometrical configurations [19].

The system excitation is rendered by the wave pressure $\Delta p^{(D)}$ calculated at the OWC inlet under the assumption of a diffracted wave field. The wave pressure $\Delta p^{(D)}$ is calculated as a function of the input wave parameters: a sinusoidal wave pressure is used in the case of regular waves, whereas for irregular waves $\Delta p^{(D)}$ is expressed as a finite sum of harmonic components whose amplitudes follow a prescribed spectral distribution [23].

\subsection{Coupling block}

A general case is studied in which the HIL setup is used to simulate OWC WECs that hold DEG PTOs which are similar to the physical prototype but present differences in several dimensional and layout features. Specifically, although the prototype system's general architecture is equivalent to the simulated system, i.e. OWC combined with DEG-PTOs made with the same materials as the hardware prototype, the following differences can be introduced: (1) the simulated OWC can feature multiple CD-DEGs connected to the same air chamber (as opposed to a single physical CD-DEG sample); (2) the simulated CD-DEGs can have larger dimensions than those of the physical samples, which are bounded by hardware constraints; (3) the simulated CD-DEGs can feature a multi-layer architecture with a different number of layers compared to the hardware setup; (4) the simulated OWC can be larger than the physical setup (e.g., it can have a larger cross section).

A consistent dynamic equivalence between the simulated and the hardware systems can be obtained by introducing a coupling block (see Fig. 2a) in the HIL scheme, which applies suitable corrections: 1) to the measured air pressure fed into the software solver and, 2) to the commanded piston position calculated based on the hydrodynamic model solution. These corrections are calculated through convenient functions of the simulation variables that are presented in this section. In particular, we propose a procedure which guarantees that the physical CD-DEG sample is subject to the same strain and electric field time history as the DEGs in the simulated scenario, assuming same time scale (e.g., operating frequencies) for the simulation and the physical experiment. In order to distinguish the variables of the simulated scenario and of the hardware setup we hereby denote the first with subscript $S$ and the latter with subscript $H$.

Since the deformed CD-DEG behaves as a thin elastomeric shell, the relative air pressure in the OWC air chamber is proportional to the CD-DEG initial thickness and the inverse of the base radius, with the proportionality factor (depending on the strain and the elastic properties) being the same in the scenario and the experiment. The following relation between the scenario and the measured air chamber pressure thus holds:

$p_{S} / p_{H}=t_{0 S} e_{H} /\left(t_{0 H} e_{S}\right)$

where $t_{0}$ and $e$ (with the appropriate subscripts) indicate the unstretched thickness and the CD-DEG's base radius (see Fig. 1b). The right hand side of Eq. (2) provides a correction factor to be applied to the measured pressure $p_{H}$ so as to obtain the pressure $p_{S}$ to be fed in the software model (i.e., into Eq. (1)).

Since the CD-DEGs strain in the experiment and in the scenario are the same, the axial displacement of the CD-DEG centre in the experiment $\left(h_{H}\right)$ and in the scenario $\left(h_{S}\right)$ simply relate as follows:

$h_{S} / h_{H}=e_{S} / e_{H}$

A relationship between the water column displacement in the scenario $z_{S}$ and the commanded piston position $z_{H}$ is then identified, assuming that the air in 
the OWC chamber undergoes adiabatic transformations. Since the chamber volume variations are small compared to the initial volume, the following linearized models for the air chamber response are assumed:

$$
\begin{gathered}
p_{S} / p_{a t m}=\gamma\left(S_{S} z_{S}-N_{S} \Omega_{S}\right) / V_{S} \\
p_{H} / p_{\text {atm }}=\gamma\left(S_{H} z_{H}-\Omega_{H}\right) / V_{H}
\end{gathered}
$$

where $p_{a t m}$ is the absolute atmospheric pressure; $\gamma$ is the air's adiabatic exponent; $V_{S}$ and $V_{H}$ are the initial air chamber volumes; $S_{S}$ is the simulated water column cross section, whereas $S_{H}$ is the hardware piston crosssection; $N_{S}$ is the number of CD-DEGs coupled with the air chamber that are devised for the simulated scenario. $\Omega_{\mathrm{S}}$ and $\Omega_{\mathrm{H}}$ are the volumes subtended by a CD-DEG in the scenario and in the experiment (positive for outward expansions), and they depend on the DEG strain and radius [6] as follows:

$\Omega_{S} / \Omega_{H}=e_{S}^{3} / e_{H}^{3}$.

Using Eqs. (2, 3, 4, 5) leads to the following relationship for the commanded piston displacement:

$z_{H}=\frac{1}{N_{S}} \frac{S_{S}}{S_{H}} \frac{e_{H}^{3}}{e_{S}^{3}} z_{S}+\left(\frac{V_{H}}{\gamma S_{H}}-\frac{t_{0 S} e_{H}^{4}}{t_{0 H} e_{S}^{4}} \frac{V_{S}}{\gamma N_{S} S_{H}}\right) \frac{p_{H}}{p_{a t m}}$

According to Eq. (6), the commanded displacement $z_{H}$ is a sum of two terms: a kinematic term proportional to the simulated water column displacement $z_{S}$, and a term which accounts for the finite air chamber compressibility [24].

Since the power density (per unit dielectric material volume) converted by a CD-DEG in a cycle depends on the strain and the applied electric field [6], we require that the DEGs in the setup and in the simulated scenario are subject to the same electric field timehistory and, hence, they generate the same electrical power density. In general, the CD-DEGs in the scenario and in the setup can have a different number of layers ( $n_{L_{S}}$ and $n_{L_{H}}$ respectively), therefore, the voltage $V_{H}$ used in the tests differs from that envisaged in the scenario $\left(V_{S}\right)$ according to the following relation, which guarantees that the electric field is an invariant:

$V_{S} / V_{H}=n_{L_{H}} t_{0 S} /\left(n_{L_{S}} t_{0 H}\right)$

Equation (7) allows selecting a suitable number of layers $n_{L_{S}}$ for the scenario, so as to limit the output voltage $V_{S}$ consistently with technical constraints.
The total power output $P_{S}$ in the simulation scenario is obtained by multiplying the measured power, $P_{H}$, by the ratio of the total dielectric material volumes in the scenario and in the physical prototype, namely:

$P_{S} / P_{H}=N_{S} e_{S}^{2} t_{0 S} /\left(e_{H}^{2} t_{0 H}\right)$

Equation (8) relies on the assumption that the CDDEG efficiency is the same in the physical prototype and in the scenario, thus neglecting scale-dependent effects. This is, in general, a conservative assumption. It is indeed expected that CD-DEGs practically applied in relevant scenarios are the result of optimised manufacturing and assembly processes and potentially feature better efficiency and timestable performance compared to the physical samples used in HIL tests.

In principle, the proposed rules allow for the simulation of DEG-based OWCs with arbitrary scale and dimensions, including the case of full-scale WECs. Compared to scaling rules for tank testing of DEG-OWCs (based on Froude scaling) [10], the proposed coupling block allows for the employment of DEG prototypes in which diameter and thickness scale factors (with respect to the scenario) are chosen independently from each other, hence granting better flexibility in terms of manufacturing. In fact, it is expected that the HIL simulator's prediction would be more reliable when the dimensions of the tested DEG prototypes are close to those in the considered scenario. HIL simulations of large-scale systems with small-scale DEGs would, in turn, suffer from errors due to an imperfect rendering of scale-sensitive effects (e.g., the DEG inertia, the electrodes resistance) which are not accounted for by the coupling block.

\subsection{Mechanical driver}

The mechanical driving system is based on a vertically mounted pneumatic cylinder that has been purposely designed and built. The system, shown in Fig. 2b, is made of a main driving cylinder-piston system with a nominal diameter of $300 \mathrm{~mm}$ (that provides $S_{H}=707$ $\mathrm{cm}^{2}$ ) and stroke of $500 \mathrm{~mm}$. The linear motion of the piston is driven by a ball-screw stage by Festo with pitch of $20 \mathrm{~mm}$ and maximum force of $4500 \mathrm{~N}$, that is actuated by an AKM52L Kollmorgen brushless motor that features rated continuous power of $2.4 \mathrm{~kW}$, 
continuous torque (at stall) of $8.67 \mathrm{Nm}$, and maximum speed of $4590 \mathrm{rpm}$. The pneumatic cylinder holds a polycarbonate cylinder at its top, on which the CDDEG assembly can be secured through a set of flanges.

Based on the volume swept by the piston and the maximum useful force (after friction losses in the O-rings), the setup can hold CD-DEGs with diameter of up to $500 \mathrm{~mm}$ and thickness of up to $0.2 \mathrm{~mm}$ after pre-stretch (i.e., $40 \mathrm{~cm}^{3}$ of dielectric material volume). Assuming convertible energy densities of $0.1-0.2 \mathrm{~J} / \mathrm{cm}^{3}$ per unit of dielectric material volume [25], noticing that a CD-DEG performs two conversion cycles during a full-oscillation of the piston (one for upward and one for downward deformation), the considered samples can produce power outputs on the order of $10^{0}-10^{1} \mathrm{~W}$ if the piston moves at a frequency of $0.2-1 \mathrm{~Hz}$. This frequency range is similar to that adopted in wave tanks for the testing of WEC prototypes at a scale between 1:30 and 1:10 (compared to a hypothetical full-scale plant installed at sea) [26]; that is, the HIL setup can be used to simulate prototypal devices over a wide dimensional range.

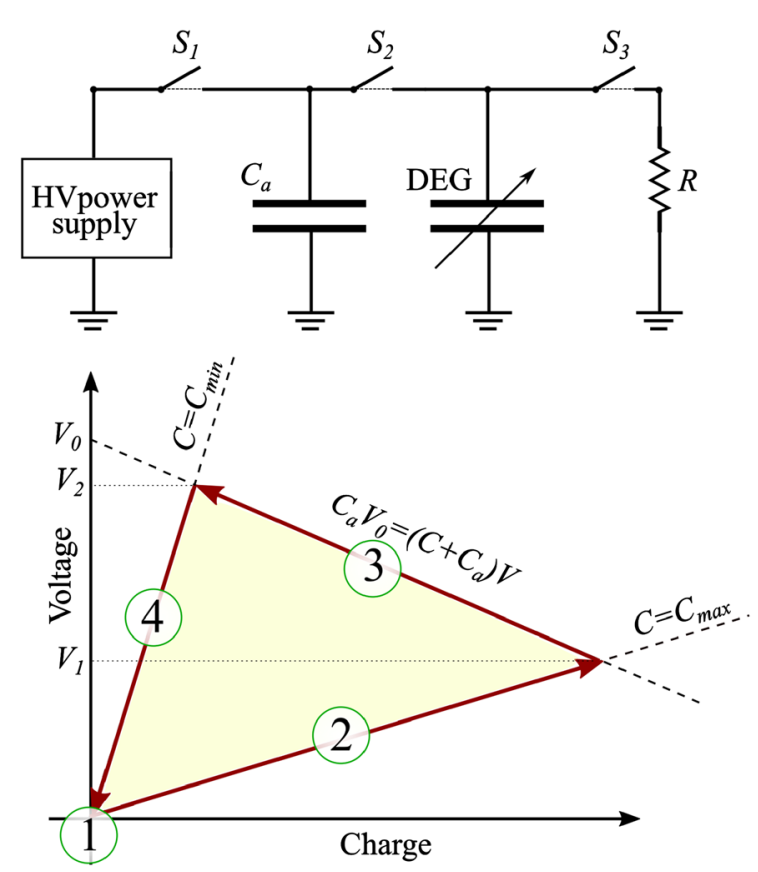

Fig. 3 Driving circuit for the CD-DEG sample and representation of the control cycle on a charge-voltage diagram
2.4 Power electronics and control logics

The control logics of DEG/OWCs is based on a prediction-free strategy, which only relies on the instantaneous measurement of the CD-DEG deformation. The control logics and power electronics used on the HIL simulator are the same as those used in [10]. A simple circuit (Fig. 3) is used, which relies on a HV amplifier (10/10B-HS by TREK), the CD-DEG sample, three HV switches (HM12-1A69-150 by MEDER Electronics), a constant capacitor $C_{a}$, and a draining resistor $R$. The circuit allows for the implementation of the following four-phase control cycle corresponding to a full oscillation of the DEG between the flat and the maximally expanded configuration:

(1) Expansion phase. During expansion (either upward or downward), the CD-DEG is kept electrically uncharged, while $C_{a}$ is charged to a fixed voltage $V_{0}$ by the power supply. During this phase, switch $S_{1}$ is kept closed, while $S_{2}$ and $S_{3}$ are open.

(2) Priming phase. When the DEG capacitance (and deformation) reaches a maximum $\left(C_{\max }\right), S_{1}$ is opened, $S_{2}$ is closed and the parallel of $C_{a}$ and the CD-DEG reaches the equilibrium voltage $V_{1}$.

(3) Generation phase. While the membrane deformation decreases, pressure makes work against the charges and mechanical energy is converted into electrostatic energy. During this phase, $S_{2}$ is kept closed, and the total charge on the DEG and $C_{a}$ holds constant (apart from leaks in the DE material).

(4) Discharging phase. Finally, when the CD-DEG reaches the maximum voltage $V_{2}$ and minimum capacitance $C_{\min }$ (i.e., in the flat position), $S_{2}$ is opened, $S_{3}$ is closed and the CD-DEG is quickly discharged through $R$.

A schematic representation of the control cycle in terms of the CD-DEG's electrical state variables (charge-voltage) is provided in Fig. 3: during phases (2) and (4) (quick charging/discharging transients) the CD-DEG position (and, hence, capacitance) holds approximately constant, whereas during phase (3) the state variables follow a trajectory which depends on the charge balance for the parallel of the CD-DEG and $C_{a}$. In practice, the capacitance might be subject to variations during phases (2) and (4), both because 
these phases are not instantaneous, and because the large voltage variations associated to charging/discharging might trigger DEG shape variations (in the same fashion as in DE actuators [27]). These variations have been shown to be negligible at the scale of the experimental CD-DEG prototypes that the HIL setup can hold, but they might become significant in case larger-scale systems are considered [10].

The use of in-parallel capacitance $C_{a}$, proposed in [25], allows for an increase of the cyclic convertible energy while limiting the voltage rise on the CD-DEG during generation phase (3). Although the proposed circuit does not enable energy storage, it makes it possible to easily estimate the net electrical energy generated in a cycle, which reads as follows:

$\mathcal{E}=\frac{1}{2} C_{\min } V_{2}^{2}-\frac{1}{2} C_{\max } V_{1}^{2}+\frac{1}{2} C_{a}\left(V_{2}^{2}-V_{1}^{2}\right)$

The first term in Eq. (9) is the energy recovered from the DEG during phase (4), the second term is the energy spent to prime the DEG, while the third term is the energy transferred to $C_{a}$ during phase (3). The maximum capacitance $C_{\max }$ reached by the CD-DEG in a cycle depends on the maximum deformation, and it can be estimated from the voltage drop on $C_{a}$ during the priming phase, namely:

$C_{\text {max }}=C_{a}\left(V_{0} / V_{1}-1\right)$

The minimum capacitance $C_{\min }$ is, in contrast, constant and it is measured in the flat CD-DEG configuration. This led the voltage $V_{2}$ to be also nearly constant (apart from minor fluctuations due to the effect of charge leaks) throughout the different cycles, with a value that is always lower than the charging voltage $V_{0}$ (see Fig. 3).

Switching from a phase to another of the control cycle is triggered based on air chamber pressure measurements. Although alternative measured or simulated state variables can be used to switch from a phase to another, pressure is used here as it is roughly proportional to DEG deformation and very easy to acquire. In particular, switching from phase (1) to phase (2) is performed based on the maxima/minima of the pressure profile; switching from phase (3) to phase (4) is triggered by the zero-crossings of the pressure; the duration of priming and discharging phases ((2), (4)) depends on characteristic charging/ discharging times set by the circuit electrical dynamics.
The power electronics layout used here was selected based on its simplicity and capability to provide a straightforward estimate of the generated electrical energy. In principle, however, this power electronics can be replaced with other advanced circuits capable, e.g., to store the generated energy rather than draining it on a resistor.

\subsection{Computation and sensing equipment}

The software environment for data acquisition, driving of the mechanical setup, and electrical control of the CD-DEG is implemented via Matlab and Simulink scripts, running the Simulink Real-Time software environment on a real-time target machine by Speedgoat at a sampling frequency of $1 \mathrm{kHz}$. Communication between the target control machine and the motor driver is handled via the EtherCAT protocol.

The voltages across the CD-DEG and $C_{a}$ are measured with two high voltage (HV) probes. The CD-DEG deformation is measured through the postprocessing of the acquired frames of a high-speed camera (Point Grey GS3-U3-23S6M-C with lens 250F6C). Specifically, the time history of the axial displacement of the central point of the membrane is obtained by following the procedure described in [8]. The piston position is monitored through the motor's integrated encoder. A pressure sensor (MPX12 by Freescale Semiconductor with custom conditioning circuit) is used to measure the pressure in the pneumatic chamber.

\section{Case study: the U-OWC plant with CD-DEG PTO}

In order to demonstrate the potential of the proposed system, we used the presented HIL framework and setup to simulate a scenario in which the CD-DEG is used as the PTO of a U-OWC WEC. The U-OWC is a special type of OWC with a U-shaped collector, provided with an inlet vertical duct, (as shown in Fig. 1.a) designed to match the device natural frequency with target wave frequencies [18].

We referred to a U-OWC pilot plant installed at NOEL laboratory of the Mediterranea University of Reggio Calabria in Italy. The reference U-OWC collector has the following dimensions (as defined in 


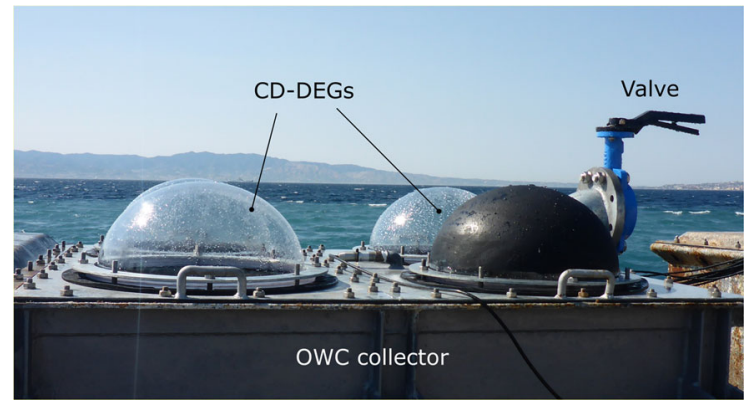

Fig. 4 U-OWC plant with CD-DEGs, tested at the NOEL in Reggio Calabria [11], the response of which is replicated through the HIL tests described here

Fig. 1a): $b_{1}=0.5 \mathrm{~m} ; b_{2}=1 \mathrm{~m} ; h_{o}=0.57 \mathrm{~m} ; l_{i}=0.8$ $\mathrm{m} ; d=1.9 \mathrm{~m}$; and a width of $1.27 \mathrm{~m}$.

The plant holds three independent chambers and it is a 1:8 scaled hardware model of a U-OWC device meant for installation in the Mediterranean Sea. In this case study we replicated a U-OWC/DEG implementation tested in the past at NOEL [11], in which one of the chambers of the plant was equipped with $N_{S}=4$ CD-DEGs made of acrylic elastomer VHB 4905 (see Fig. 4). Those sea tests were aimed at studying only the passive mechanical response of the U-OWC/DEG system, therefore, no electrical activation was provided to the membranes. Here, in contrast, we implemented an electrically functional CD-DEG prototype and investigated the fully-coupled hydro-electro-viscoelastic response of the system in different sea conditions. The considered PTO is made of dielectric acrylic VHB 4905, which was chosen as the DE material because of its ease of handling, pre-stretching and stacking. We assumed that the physical CD-DEG sample has the same base radius $\left(e_{H}=e_{S}=0.195 \mathrm{~m}\right)$ and pre-stretch $\left(\lambda_{p}=3.44\right)$ as the CD-DEGs in the scenario. We further assumed that the CD-DEGs in the scenario have an unstretched thickness $t_{0 S}=5 \mathrm{~mm}$ (0.42 mm after pre-stretch), whereas the physical sample has unstretched thickness $t_{0 H}=3 \mathrm{~mm}$ $(0.25 \mathrm{~mm}$ after pre-stretch). This choice allowed using electric fields in the order of $10^{1}-10^{2} \mathrm{kV} / \mathrm{mm}$ (consistent with the breakdown limits of the reference elastomer [25]) with a limited number of DE layers in the stack and operating voltages below $10 \mathrm{kV}$.

Compared to the sea tests in [11], for the purpose of the HIL test-bench demonstration, here we considered a set of milder sea states. We focused on operating conditions in which the OWC air chamber is held closed (whereas in [11] a safety valve, held partly open, was present, as shown in Fig. 4) and we simulated its response in the presence of both regular (sinusoidal) and irregular (stochastic) [23] wave excitation, with wave heights of $5-15 \mathrm{~cm}$ and wave periods up to $3.5 \mathrm{~s}$. Although these sea states are rather mild with respect to typical conditions at the NOEL test site, they allow safe operation of the CD-DEGs, thus representing reasonable realistic conditions for the implementation of pilot sea tests with fullyfunctional DEGs in the near future. To enable operation in the presence of more energetic sea states, the reference U-OWC should be equipped with CDDEGs featuring larger diameter and thickness, made of more reliable materials (e.g., silicone dielectric with carbon-loaded silicone electrodes [28]). This would require further effort in terms of manufacturing and power electronics implementation, and hence appears an experimentally viable scenario only in a longerterm perspective.

\subsection{Hydrodynamic model and coupling}

The U-OWC oscillation $z_{S}$ is governed by Eq. (1), with the mass $M(z)$ and the damping $C(z, \dot{z})$ terms taking the following specific forms [19]:

$$
\begin{array}{r}
M\left(z_{S}\right)=\frac{1+C_{\text {in }}}{g}\left(\frac{b_{2}}{b_{1}} l_{i}+l_{i}+h+z_{S}\right)+\frac{b_{2}}{g b_{1}} A(\infty) \\
C\left(z_{S}, \dot{z}_{S}\right)=\frac{1}{2 g}\left\{1+C_{d g}\left[\frac{l_{i}}{R_{h 1}}\left(\frac{b_{2}}{b_{1}}\right)^{2}+\frac{l_{i}+h+z_{S}}{R_{h 2}}\right]\right\}|\dot{z}|
\end{array}
$$

Compared to the model in Sect. 2.1, additional terms are here considered which account for hydraulic head losses according to the so-called instantaneous acceleration based model [29]. $C_{i n}=0.13$ and $C_{d g}=$ 0.71 in Eq. (11), are empirical coefficients [29]. $R_{\mathrm{h} 1}$ and $R_{\mathrm{h} 2}$ are called hydraulic radii of the vertical duct and of the chamber respectively [29], and they are defined as follows:

$R_{h 1}=\frac{b_{1} b_{3}}{2\left(b_{1}+b_{3}\right)}, R_{h 2}=\frac{b_{2} b_{3}}{2\left(b_{2}+b_{3}\right)}$

The hydrodynamic parameters $(A(\infty), K(t))$ and the excitation pressure as a function of the wave parameters are computed based on the analytical model presented in [19]. 
As regards coupling of the simulation and the physical variables, Eq. (6) requires the piston position $z_{H}$ to be commanded as a function of the simulated water column displacement $z_{S}$ and the measured pressure, $p_{H}$, which is naturally affected by noise. The presence of a moderate noise on $p_{H}$ does not affect the calculation of $z_{S}$, since the solution of Eq. (1) involves a double integration operation, which results in a smooth profile for $z_{S}$. In contrast to that, an additional term proportional to $p_{H}$ in the expression of $z_{H}$ (Eq. (6)) can lead to vibrations (which are especially critical because of the friction between piston and cylinder) or to close-loop instability in case signal filtering is applied. To overcome this issue and for the sake of simplicity, we chose to replace the measured pressure in Eq. (6) with a static averaged experimental trend of the air chamber pressure, $p_{H}=\tilde{\mathrm{p}}_{H}\left(z_{H}\right)$, hence expressing $z_{H}$ solely as a function of $z_{S}$. This choice granted the achievement of a smooth motion of the piston (regardless of pressure signal filtering quality), while introducing a limited error.

\subsection{CD-DEG sample and power electronics}

The CD-DEG has been built using the procedure introduced in [10]. The sample enjoys a multi-layer structure (see Fig. 1.b) with two in-parallel dielectric layers separated by three carbon grease (MG-Chemicals 846) electrodes: two ground electrodes on the outer faces of the stack, and a HV electrode in the middle. Each dielectric layer $(1.5 \mathrm{~mm}$ thick before pre-stretch) has been obtained by bonding three VHB 4905 layers together.

The CD-DEG sample's measured capacitance $C_{\min }$ in the flat equilibrium configuration was $76.5 \mathrm{nF}$. The DEG was driven via the circuit and control logics described in Sect. 2.4, using an in-parallel capacitance $C_{a}=300 \mathrm{nF}$, and draining resistance $R=100 \mathrm{k} \Omega$. A voltage $V_{0}=9 \mathrm{kV}$ was used to prime $C_{a}$, leading to maximum electric fields on the CD-DEG in the order of $130 \mathrm{kV} / \mathrm{mm}$, similar to [10].

\subsection{HIL setup validation}

We performed a set of tests to validate the HIL setup and assess its robustness against possible latencies, delays in the control loop, and simplifications due to the coupling criteria presented in Sect. 2.2. Similarly to [12], we assessed the ability of the HIL framework and setup to reproduce the results of a reference hydroelastic model of the U-OWC and the DEG PTO. The target model, presented and validated in [11] via sea tests, relies on the same hydrodynamic model (validated, e.g., in [29]) used in the present HIL formulation and on a numerical model of the CD-DEG. Compared to [11], in the reference model we slightly updated the CD-DEG elastic parameters in order to better capture the pressure-deformation response of the sample under investigation, so as to mitigate the effect of the elastic parameters uncertainty. We compared the oscillation amplitudes of some relevant variables for the target model and the HIL setup in a set of regular wave tests with periods between $1.5 \mathrm{~s}$ and $3.5 \mathrm{~s}$ spaced apart by $0.5 \mathrm{~s}$. Figure 5 compares the oscillation amplitudes (HIL simulations vs. reference model) of the water column displacement (Fig. 5a) and the relative air pressure (Fig. $5 \mathrm{~b}$ ) for a wave height of $50 \mathrm{~mm}$. HIL data in the plots are projected to the scenario scale using the rules defined in Sect. 2.2. The plots show that the HIL framework faithfully replicates the trend and the amplitudes of the model, hence proving the robustness and stability of the closed-loop coupling of the software model and the physical setup. Mismatches between the model and the HIL data in terms of the free surface elevation data are larger than those in the air pressure data (e.g., at $0.5 \mathrm{~Hz}$, the model overestimates the free surface elevation, while
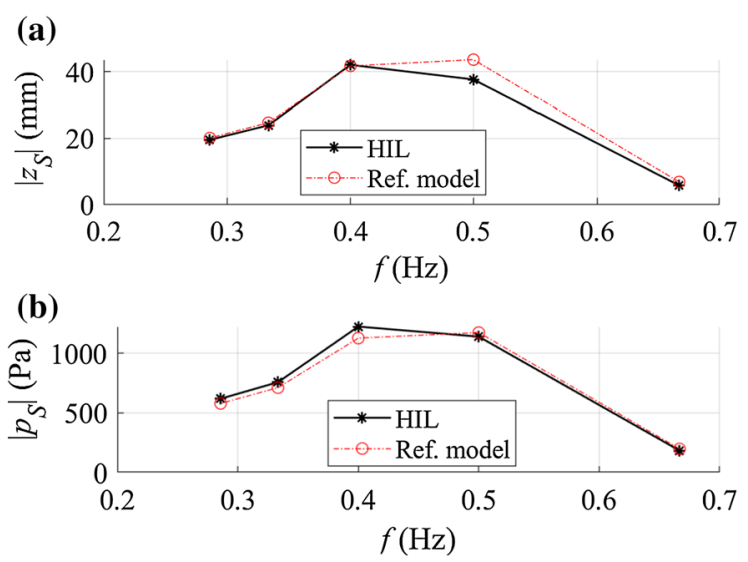

Fig. 5 Validation of the HIL setup against a reference U-OWC/ DEG model [11]. Comparison of oscillation amplitudes of a water column oscillations, b air chamber pressure in a set of monochromatic tests with height $H=50 \mathrm{~mm}$ at different frequencies 
reasonably capturing the pressure). This is ascribable to inaccuracies in the CD-DEG model, which affects the relationship between water surface and pressure. In fact, the CD-DEG model relies on strong simplifications, and is thus prone to errors, which are not presented in the HIL simulator, where the CD-DEG model is replaced with a physical prototype [30].

Interestingly, the mismatch between the reference model and the HIL results higher at wave frequencies of $0.4-0.5 \mathrm{~Hz}$, where the oscillation amplitudes are at their maximum (i.e., the system operates at the mechanical resonance). This is most probably due to the CD-DEG viscous dissipations, which are not accounted for in the target model and can influence the system response in the presence of large water column oscillations and velocities typical of the resonance condition. This means that the accurate description of the response of the system at resonance via a mathematical model is rather difficult. At the same time, the behaviour at resonance is of high interest, since this is the condition at which the best power/efficiency performance is recorded. This, hence, offers a strong motivation towards the employment of a HIL approach as opposed to purely numerical models.

\subsection{Results}

The HIL setup and framework has been used to characterise the response of the reference U-OWC/ DEG system in different wave conditions. Specifically, two datasets illustrating the functionality of the HIL test-bench are shown in Fig. 6. The plots show portions of time-series of the measured variables
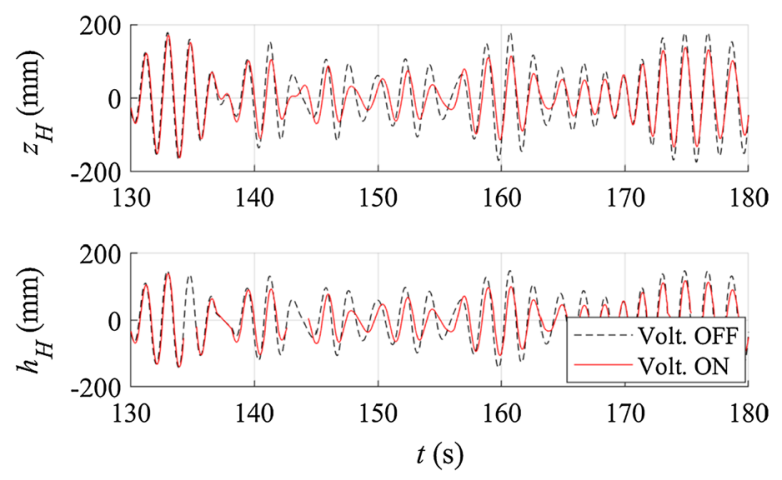

Fig. 6 Time-series showing the HIL test-bench functionality. The datasets refer to an irregular sea state with JONSWAP spectral distribution, significant wave height $H_{s}=150 \mathrm{~mm}$, peak period $T_{p}=1.65 \mathrm{~s}$. Dashed lines refer to a passive (piston position $z_{H}$, air chamber pressure $p_{H}$, membrane tip displacement $h_{H}$, and voltage $V_{H}$ ) in two tests in which the simulated U-OWC was subject to incident irregular waves compatible with a JONSWAP frequency spectrum [23], similar to the actual sea states experimentally observed at NOEL [31]. The plot refers to a sea state with significant wave height $H_{S}=$ $0.15 \mathrm{~m}$, peak period $T_{p}=1.65 \mathrm{~s}$ and peak enhancement factor of 3.3, which is representative of a mild sea condition at NOEL. The figure compares the results of two test runs: 1) a passive test with no voltage applied on the DEG; and 2) a power generation test, with electrically controlled DEG. The two datasets present an initial overlap, as no voltage is initially applied on the DEG in both tests. Following that, the application of a voltage on the DEG visibly affects the system dynamics, leading to a reduction in the average amplitude of the oscillations, mainly due to the CD-DEG PTO damping. As shown by the voltage time-series, the voltage $V_{1}$ reached by the CDDEG after priming changes at each cycle, owing to the different deformation amplitudes reached, whereas the maximum voltage $V_{2}$ is approximately the same (i.e., around $7 \mathrm{kV}$ ) for the different cycles (excluding the initial transient), as observed in Sect. 2.4. The controller was programmed to prevent the activation of the CD-DEG during certain cycles in which the deformation was small (namely, the measured pressure module is below $200 \mathrm{~Pa}$ ), since in those situations the electrical losses might exceed the generated energy. In the power generation test, the average generated power in this test was calculated as the sum of the energies over the different cycles (Eq. (9))
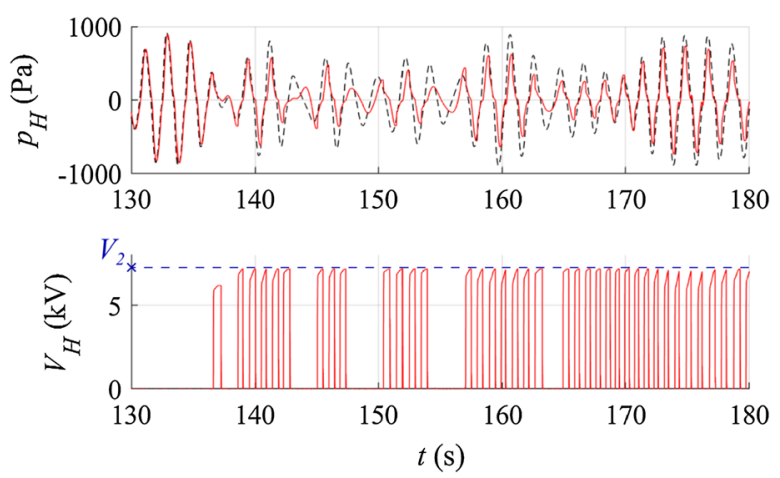

mechanical test, whereas solid lines refer to a test with active electrical control 
divided by the total duration of the test. The average generated power (over a time window of $120 \mathrm{~s}$ ) was $0.5 \mathrm{~W}$, with peaks of up to $1.2 \mathrm{~W}$ (calculated from the generated energy and the duration of the cycles with maximum deformation). Based on Eq. (8), this renders a power of $3.2 \mathrm{~W}$ (with peaks of $7.7 \mathrm{~W}$ ) in the considered scenario, i.e. a significant fraction (over $15 \%$ in average) of the power available in the considered mild sea state.

Following that, we performed a systematic study of the coupled U-OWC/DEG dynamic response through regular wave tests. These tests are an effective tool to investigate the system frequency response [10], and they provide useful information that would be unavailable via sea tests with purely stochastic excitation. Consistently with the hardware setup limitations, we ran regular wave tests using a constant wave height $H=50 \mathrm{~mm}$ and wave periods within the typical frequency range at NOEL. Each regular wave test comprised a first phase in which the electrical control was not active (namely, no voltage was present on the CD-DEG sample), and a second phase in which the DEG was actively controlled. This allowed for a characterisation of the system steady-state mechanical passive response and the electrically active response.

Some relevant experimental time-series relative to two regular wave tests are shown in Fig. 7. In particular, the dataset in Fig. 7.a refers to a wave period $T=2.5 \mathrm{~s}$ (frequency $f=0.4 \mathrm{~Hz}$ ), i.e., close to the system natural frequency (as observed in Sect. 3.3), whereas the dataset in Fig. 7.b refers to a wave period $T=3.5 \mathrm{~s}$ (frequency $f=0.29 \mathrm{~Hz}$ ), i.e. below the system natural frequency. Applying electrical activation on the U-OWC/DEG has the double

(a)
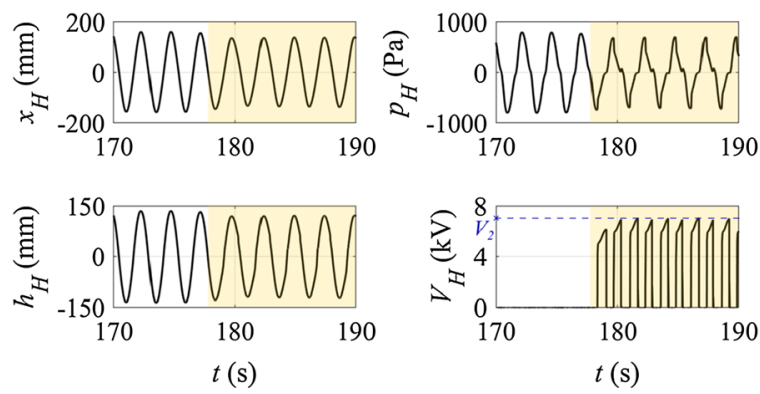

Fig. 7 Experimental time-series of water column displacement, air pressure, membrane centre displacement, and CD-DEG voltage for two regular wave sea states: a $H=50 \mathrm{~mm}, T=2.5$ effect of causing a decrease in the CD-DEG stiffness and damping the system oscillations. As a consequence:

- The oscillation amplitudes of the water column, pressure, membrane displacement increase upon electrical activation when the wave frequency is below the plant natural frequency (Fig. 7b). This is due to the electrically-induced softening of the DE layers, which causes the system natural frequency to decrease, hence approaching the excitation frequency.

- At an excitation frequency close to the natural frequency (Fig. 7a), oscillation amplitudes decrease because of the electrical activation, as the result of a reduction in the system natural frequency compared to the excitation frequency, and of electrical damping.

The air chamber pressure time-series further show visible drops in correspondence of the charging transients, as a result of the CD-DEG stiffness decrease due to electrical activation. The oscillatory behavior of the pressure in correspondence of the zero crossings is ascribable to a partial loss of tension in the membrane, possibly due to viscoelasticity.

An overview of regular wave test results is presented in Fig. 8, which shows the oscillation amplitudes of water column position, air pressure, and CD-DEG tip position (at the scale of the scenario) for the passive mechanical case and the electrically active case. The natural frequency in the presence of electrical activation slightly decreases (although in Fig. 8 the maxima stay at $0.4 \mathrm{~Hz}$, because of the coarse resolution of the chosen frequency grid) and the (b)
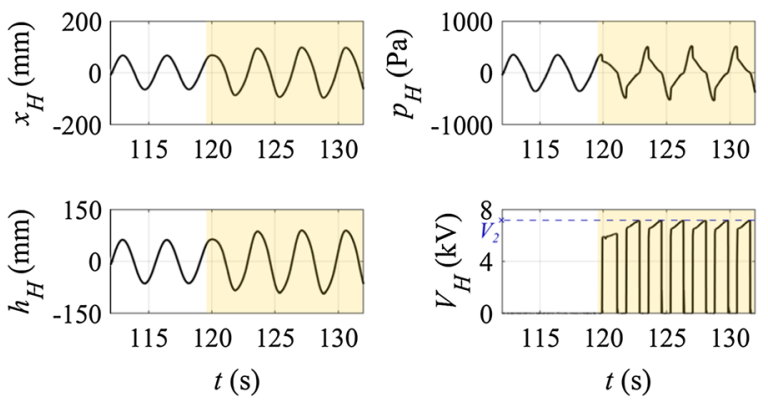

s, b $H=50 \mathrm{~mm}, T=3.5 \mathrm{~s}$. Coloured areas highlight the phases during which electrical control is switched on 
(a)

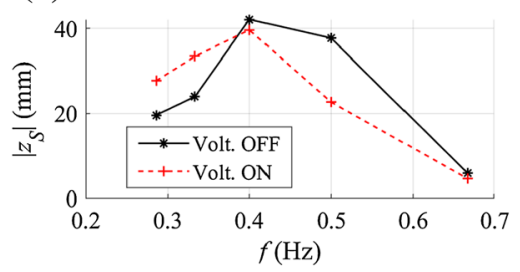

(b)

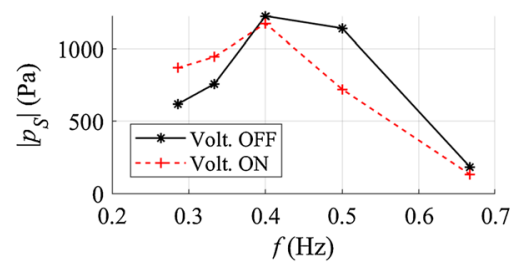

(c)

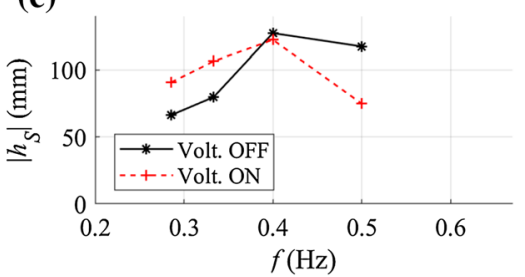

Fig. 8 Regular wave test results (wave height $H=50 \mathrm{~mm}$ ). a Water column displacement, b air pressure, and $\mathbf{c}$ membrane centre displacement oscillation amplitudes with and without electrical activation. Different markers refer to different tests with excitation period $T$ between $1.5 \mathrm{~s}$ and $3.5 \mathrm{~s}$ (spaced apart

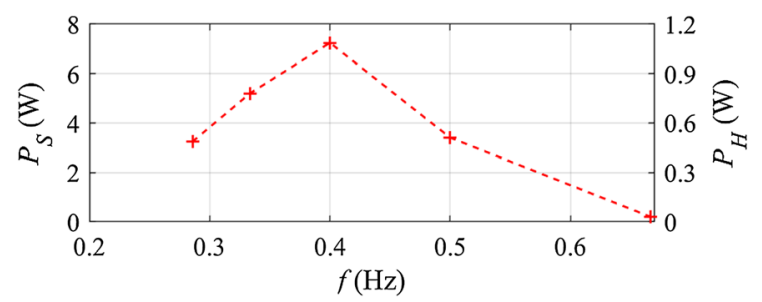

Fig. 9 Generated electrical power for simulated scenario (left axis), and experimental (right axis) in regular wave tests with wave height $H=50 \mathrm{~mm}$

oscillation amplitudes increase (decrease) at low (high) frequencies upon control application, because of electrically-induced variations in the system natural frequency. The peak values in the water column motion are lower in the presence of electrical activation, as a result of the PTO damping.

The power $P_{H}$ generated by the CD-DEG sample and the corresponding power output $P_{S}$ of the U-OWC plant are shown in Fig. 9. The generated power $P_{H}$ is estimated from the average energy generated during the different cycles (Eq. (9)), whereas $P_{S}$ is calculated using Eq. (8). The CD-DEG samples generated powers over $1 \mathrm{~W}$, corresponding to a few Watts at the scenario scale. This is a rather small power considering the dimensions of the plant, but it represents a significant fraction of the input power of the mild incident waves considered in these tests. The present results hence confirm the effectiveness of the proposed control strategy for the CD-DEG and effectiveness of the DEG PTO in combination with a dynamical system and broadband mechanical power sources. Larger powers could be obtained (both in the scenario and with the HIL test-bench) by selecting DEGs with a larger thickness (subject to more energetic sea states), at the expense of a greater experimental burden. by $0.5 \mathrm{~s}$ ). The DEG oscillation amplitudes at $f=0.67 \mathrm{~Hz}$ ( $T=1.5 \mathrm{~s}$ ) are not available: because of the small deformations, the membrane was hidden by the support structure and could not be detected through the camera frames

The present results are encouraging in view of future sea tests implementing the scenario simulated in this article. Such tests are indeed feasible with the simplified manufacturing technology used here and in previous works [10] and with simple laboratory-scale electronics. These results suggest that, by selecting a suitable window of mild sea conditions, it would be possible to achieve power generation from the waves up to nearly $10 \mathrm{~W}$. Despite representing a modest energy income in relation to the target U-OWC WEC, this would represent the largest power generation test ever realized with DEGs, and would pave the way to further research on DEG PTOs scaling-up via improved electronics, materials and manufacturing strategies.

In addition to that, the HIL setup might be employed in the simulation of larger scale systems, e.g., the full-scale DEG-based U-OWC design envisaged in [11]. In principle, this full-scale WEC can be simulated with the presented HIL setup, by simply relying on the coupling laws discussed in Sect. 2.2. In practice, this would require the use of a different DE material compared to the leaky and unreliable acrylic used in this study.

\section{Conclusions}

This paper presents a hardware-in-the-loop (HIL) framework and setup to simulate sea wave energy converters (WECs) equipped with dielectric elastomer generator (DEG) power take-off (PTO) systems. Attention is focused on a particular class of WECs, namely, the oscillating water column (OWC). DEGbased OWCs represent a promising upcoming class of 
low-cost and efficient WECs, which have been increasingly investigated in the recent past.

The proposed HIL framework relies on a software model of OWCs' hydrodynamics, coupled, in a closed-loop fashion, with a physical prototype of a pneumatically-driven circular diaphragm DEG (CDDEG), and its electro-mechanical driving interface. The hydrodynamic model solves in real-time the dynamic equation of motion for the OWC plant based on simulated wave-induced loads. The solution is used to pilot the mechanical hardware that drives the deformation of the CD-DEG. The energy harvesting cycle is implemented at hardware level, through a purposely developed high voltage electronic driver that implements simple control logics based on air chamber pressure measurement. The actual pneumatic load measured on the CD-DEG is in turn fed into the software model and accounted for in the simulated dynamics.

Compared to wave tank or sea tests, this HIL framework allows operating in a controlled laboratory environment, hence drastically reducing the experimental burden and cost while ensuring an exact rendering of the DEG PTO response and performance. The framework thus provides a powerful tool to test different CD-DEGs implementations and control strategies subject to realistic operating conditions.

We applied the HIL setup to study the behaviour of a particular OWC/DEG implementation, based on the so-called U-OWC layout. Building upon previous sea tests, we characterised the response of a small-scale U-OWC plant equipped with a fully-functional CDDEG PTO, highlighting the features of the system dynamical response. Besides demonstrating the effectiveness of the proposed HIL framework, we obtained results that provide relevant indications for future sea tests and further upscaling of the DEG PTO technology for wave energy conversion.

Acknowledgement The authors would like to thank Dr. G. P. Rosati Papini (University of Trento) who offered support with the high-speed camera video processing and Eng. Francesco Damiani (Cheros Engineering Srl) who offered his support with the high voltage circuit design.

Funding Giacomo Moretti received funds from the European Union's Horizon 2020 research and innovation programme under the Marie Skłodowska-Curie grant agreement No 893674 (DEtune). Open access funding provided by Scuola Superiore Sant'Anna within the CRUI-CARE Agreement.

\section{Compliance with ethical standards}

Conflict of interest The authors declare that they have no conflict of interest.

Open Access This article is licensed under a Creative Commons Attribution 4.0 International License, which permits use, sharing, adaptation, distribution and reproduction in any medium or format, as long as you give appropriate credit to the original author(s) and the source, provide a link to the Creative Commons licence, and indicate if changes were made. The images or other third party material in this article are included in the article's Creative Commons licence, unless indicated otherwise in a credit line to the material. If material is not included in the article's Creative Commons licence and your intended use is not permitted by statutory regulation or exceeds the permitted use, you will need to obtain permission directly from the copyright holder. To view a copy of this licence, visit http://creativecommons.org/licenses/by/4.0/.

\section{References}

1. Pecher A, Kofoed JP (2017) Handbook of ocean wave energy. Springer, Cham

2. Chiba S, Waki M (2020) Innovative power generator using dielectric elastomers (creating the foundations of an environmentally sustainable society). Sustain Chem Pharm. 15:100205. https://doi.org/10.1016/j.scp.2019.100205

3. Kaltseis R et al (2014) Natural rubber for sustainable highpower electrical energy generation. RSC Adv 4(53):27905. https://doi.org/10.1039/c4ra03090g

4. Carpi F et al (2015) Standards for dielectric elastomer transducers. Smart Mater Struct 24(10):105025. https://doi. org/10.1088/0964-1726/24/10/105025

5. Moretti G, Rosset S, Vertechy R, Anderson I, Fontana M (2020) A review of dielectric elastomer generator systems. Adv Intell Syst 2(10):2000125. https://doi.org/10.1002/ aisy. 202000125

6. Moretti G et al (2020) Advances in the development of dielectric elastomer generators for wave energy conversion. Renew Sustain Energy Rev. https://doi.org/10.1016/j.rser. 2019.109430

7. Heat TV (2012) A review of oscillating water columns. Philos Trans R Soc A Math Phys Eng Sci 370:235-245. https://doi.org/10.1098/rsta.2011.0164

8. Falcão AFO, Henriques JCC (2016) Oscillating-water-column wave energy converters and air turbines: a review. Renew Energy 85:1391-1424. https://doi.org/10.1016/j. renene.2015.07.086

9. Moretti G, Righi M, Vertechy R, Fontana M (2017) Fabrication and test of an inflated circular diaphragm dielectric elastomer generator based on PDMS rubber composite. Polymers 9(7):283. https://doi.org/10.3390/polym9070283

10. Moretti $G$ et al (2019) Modelling and testing of a wave energy converter based on dielectric elastomer generators. Proc R Soc A Math Phys Eng Sci. https://doi.org/10.1098/ rspa.2018.0566

11. Moretti $\mathrm{G}$ et al (2020) Modelling and field testing of a breakwater-integrated U-OWC wave energy converter with 
dielectric elastomer generator. Renew Energy. https://doi. org/10.1016/j.renene.2019.06.077

12. Signorelli C, Villegas C, Ringwood J (2011) Hardware-inthe-loop simulation of a heaving wave energy converter. In: Proceedings of the 9th European wave and tidal energy conference, Southampton, UK, 5-9 September

13. De Falcão AFO, Pereira PER, Henriques JCC, Gato LMC (2010) Hydrodynamic simulation of a floating wave energy converter by a U-tube rig for power take-off testing. Ocean Eng 37(14-15):1253-1260. https://doi.org/10.1016/j. oceaneng.2010.05.007

14. Bracco G, Giorcelli E, Mattiazzo G, Orlando V, Raffero M (2015) Hardware-in-the-loop test rig for the ISWEC wave energy system. Mechatronics 25:11-17. https://doi.org/10. 1016/J.MECHATRONICS.2014.10.007

15. Rea J, Kelly J, Alcorn R, O'Sullivan D (2011) Development and operation of a power take off rig for ocean energy research and testing. In: Proceedings of the 9th European wave and tidal energy conference, Southhampton, UK, 5-9 September

16. Kelly JF, Christie R (2017) Applying hardware-in-the-loop capabilities to an ocean renewable energy device emulator. Twelfth international conference on ecological vehicles and renewable energies (EVER), Apr. 2017, pp. 1-7, doi: https://doi.org/10.1109/EVER.2017.7935943

17. Moretti G, Rosati GPP, Fontana M, R. Vertechy R (2015) Hardware in the loop simulation of a dielectric elastomer generator for oscillating water column wave energy converters. In: OCEANS 2015 - Genova, May 2015, pp. 1-7, doi: $\quad$ https://doi.org/10.1109/OCEANS-Genova.2015. 7271571

18. Boccotti P, Filianoti P, Fiamma V, Arena F (2007) Caisson breakwaters embodying an OWC with a small opening-Part I: theory. Ocean Eng 34(5-6):820-841. https://doi.org/10. 1016/j.oceaneng.2006.04.016

19. Malara G, Gomes RPF, Arena F, Henriques JCC, Gato LMC, Falcão AFO, (2017) The influence of three-dimensional effects on the performance of U-type oscillating water column wave energy harvesters. Renew Energy 111:506-522. https://doi.org/10.1016/j.renene.2017.04.038

20. Mei CC, Stiassnie M, Yue DKK (2005) Theory and applications of ocean surface waves, vol 23. World Scientific, Singapore

21. Cummins WE (1962) The impulse response function and ship motions. Schiffstechnik 57(9):101-109
22. Newman JN, Lee C-H (2002) Boundary-element methods in offshore structure analysis. J Offshore Mech Arct Eng 124(2):81. https://doi.org/10.1115/1.1464561

23. Boccotti P (2000) Wave mechanics for ocean engineering. Elsevier Oceanography Series, Amsterdam, The Netherlands

24. Falcão AFO, Henriques JCC (2019) The spring-like air compressibility effect in oscillating-water-column wave energy converters: review and analyses. Renew Sustain Energy Rev 112:483-498. https://doi.org/10.1016/J.RSER. 2019.04.040

25. Shian S, Huang J, Zhu S, Clarke DR (2014) Optimizing the electrical energy conversion cycle of dielectric elastomer generators. Adv Mater 26(38):6617-6621. https://doi.org/ 10.1002/adma.201402291

26. Ingram D, Wallace R, Robinson A, Bryden I (2014) The design and commissioning of the first, circular, combined current and wave test basin. In: Oceans 2014 MTS/IEEE, 2014, doi: https://doi.org/10.1109/OCEANS-TAIPEI.2014. 6964577

27. Zanini P, Rossiter J, Homer M (2015) Modelling the effect of actuator-like behavior in dielectric elastomer generators. Appl Phys Lett 107(15):153906. https://doi.org/10.1063/1. 4933315

28. Madsen FB, Daugaard AE, Hvilsted S, Skov AL (2016) The current state of silicone-based dielectric elastomer transducers. Macromol Rapid Commun 37(5):378-413. https:// doi.org/10.1002/marc.201500576

29. Malara G, Romolo A, Fiamma V, Arena F (2017) On the modelling of water column oscillations in U-OWC energy harvesters. Renew Energy 101:964-972. https://doi.org/10. 1016/j.renene.2016.09.051

30. Vertechy R et al (2014) Reduced model and application of inflating circular diaphragm dielectric elastomer generators for wave energy harvesting. J Vib Acoust 137(1):011016. https://doi.org/10.1115/1.4028508

31. Arena F, Barbaro G (2013) The natural ocean engineering laboratory, NOEL, in Reggio Calabria, Italy: a commentary and announcement. J Coast Res. https://doi.org/10.2112/ 13A-00004

Publisher's Note Springer Nature remains neutral with regard to jurisdictional claims in published maps and institutional affiliations. 